\title{
Trifluoroacetic Acid and Trifluoroacetic Anhydride Radical Cations Dissociate Near
}

\section{the Ionization Limit}

Lukas Lesniak, a Juana Salas, ${ }^{\mathrm{b}}$ Jake Burner, ${ }^{\mathrm{a}}$ Malick Diedhiou, ${ }^{\mathrm{a}}$ Maxi A Burgos Paci, ${ }^{\mathrm{b}}$ Andras Bodic and Paul M Mayer ${ }^{\mathrm{a}^{*}}$

a Department of Chemistry and Biomolecular Sciences, University of Ottawa, Ottawa, Canada K1N 6N5

b INFIQC - CONICET, Departamento Fisicoquimica, Universidad Nacional de Córdoba, Cordoba, Argentina

c Paul Sherrer Institute, Villigen, 5232 Switzerland

*Corresponding author: Paul M Mayer, Department of Chemistry and Biomolecular Sciences, University of Ottawa, 10 Marie Curie, Ottawa, Canada K1N 6N5. pmmayer@uottawa.ca

Supporting Information

\section{Reference 25}

Frisch, M. J.; Trucks, G. W.; Schlegel, H. B.; Scuseria, G. E.; Robb, M. A.; Cheeseman, J. R.; Scalmani, G.; Barone, V.; Petersson, G. A.; Nakatsuji, H.; Li, X.; Caricato, M.; Marenich, A. V.; Bloino, J.; Janesko, B. G.; Gomperts, R.; Mennucci, B.; Hratchian, H. P.; Ortiz, J. V.; Izmaylov, A. F.; Sonnenberg, J. L.; Williams; Ding, F.; Lipparini, F.; Egidi, F.; Goings, J.; Peng, B.; Petrone, A.; Henderson, T.; Ranasinghe, D.; Zakrzewski, V. G.; Gao, J.; Rega, N.; Zheng, G.; Liang, W.; Hada, M.; Ehara, M.; Toyota, K.; Fukuda, R.; Hasegawa, J.; Ishida, M.; Nakajima, T.; Honda, Y.; Kitao, O.; Nakai, H.; Vreven, T.; Throssell, K.; Montgomery Jr., J. A.; Peralta, J. E.; Ogliaro, F.; Bearpark, M. J.; Heyd, J. J.; Brothers, E. N.; Kudin, K. N.; Staroverov, V. N.; Keith, T. A.; Kobayashi, R.; Normand, J.; Raghavachari, K.; Rendell, A. P.; Burant, J. C.; Iyengar, S. S.; Tomasi, J.; Cossi, M.; Millam, J. M.; Klene, M.; Adamo, C.; Cammi, R.; Ochterski, J. W.; Martin, R. L.; Morokuma, K.; Farkas, O.; Foresman, J. B.; Fox, D. J., Gaussian 16, Wallingford, CT, 2016. 
Figure S1. Comparison of the TPES derived from effusive and molecular beam sample introduction.

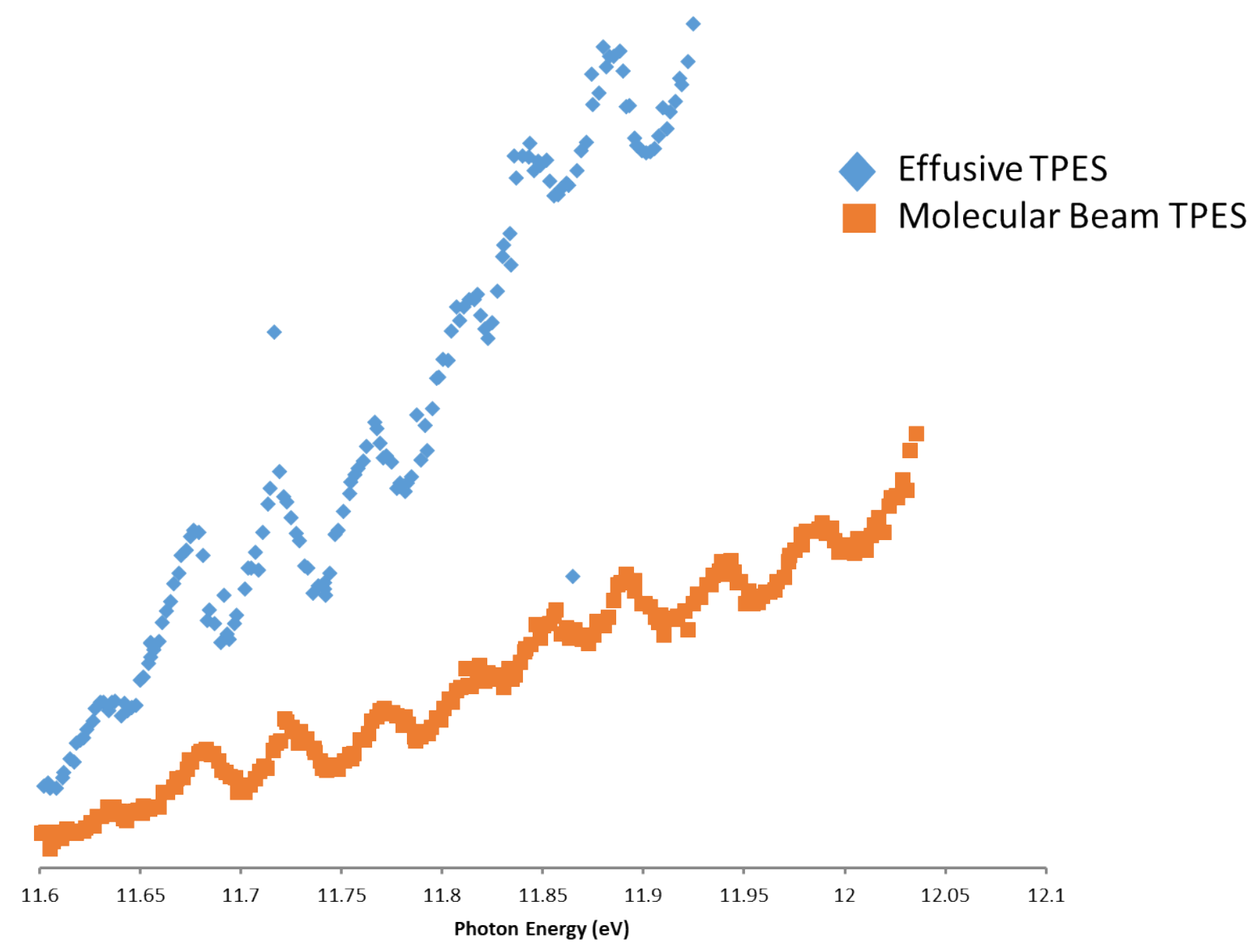

